INPLASY

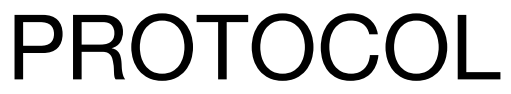

To cite: Ye et al. The Effect of Traditional Chinese Exercise for Knee Osteoarthritis: A study protocol for a systematic review and meta-analysis. Inplasy protocol 202080109. doi:

10.37766/inplasy2020.8.0109

Received: 26 August 2020

Published: 27 August 2020

Corresponding author:

Qing-qing Ye

18408203982@163.com

Author Affiliation:

Yibin Hospital of Traditional

Chinese Medicine

Support: None

Review Stage at time of this submission: Preliminary

searches.

Conflicts of interest:

None.

\section{The Effect of Traditional Chinese Exercise for Knee Osteoarthritis: A study protocol for a systematic review and meta-analysis}

\author{
Ye, QQ1; Liu, L2; Zhang, F3; Yang, F4; Shi, XY5; Liu, B6.
}

Review question / Objective: Traditional Chinese Exercise (TCE) is an indispensable form of Traditional Chinese Medicine, including a variety of exercise, such as Qigong, Tai Chi, Baduanjin, which has been recognized to effectively relieve chronic pain in patients with knee osteoarthritis(KOA), but the evidence for its effect(benefits and harms) is still controversial.

Condition being studied: Knee Osteoarthritis (KOA) is becoming one of the most common joint condition troubled the middle-aged and elderly as a result of the increased life expectancy and ageing of the global population, it is becoming an increasingly major socioeconomic and public health issue. Knee Osteoarthritis (KOA) is becoming one of the most common joint condition troubled the middle-aged and elderly as a result of the increased life expectancy and ageing of the global population, it is becoming an increasingly major socioeconomic and public health issue. These findings will provide guidance to clinicians and patients on the use of TCE.

INPLASY registration number: This protocol was registered with the International Platform of Registered Systematic Review and Meta-Analysis Protocols (INPLASY) on 27 August 2020 and was last updated on 27 August 2020 (registration number INPLASY202080109).

\section{INTRODUCTION}

Review question / Objective: Traditional Chinese Exercise (TCE) is an indispensable form of Traditional Chinese Medicine, including a variety of exercise, such as Qigong, Tai Chi, Baduanjin, which has been recognized to effectively relieve chronic pain in patients with knee osteoarthritis(KOA), but the evidence for its effect(benefits and harms) is still controversial.

Rationale: For thousands of years in ancient China, traditional Chinese exercise (TCE), regarded as the typical form of 
meditative movement that uses physical activity to harmonize the body, mind and spirit, has been widely practised in China.It can achive the purpose of mobilising $Q i$ and blood coordinately, and maintaining the balance of Yin and Yang(the two opposite properties of all natural things in traditional Chinese medicine) of human.

Condition being studied: Knee Osteoarthritis (KOA) is becoming one of the most common joint condition troubled the middle-aged and elderly as a result of the increased life expectancy and ageing of the global population, it is becoming an increasingly major socioeconomic and public health issue. Knee Osteoarthritis (KOA) is becoming one of the most common joint condition troubled the middle-aged and elderly as a result of the increased life expectancy and ageing of the global population, it is becoming an increasingly major socioeconomic and public health issue. These findings will provide guidance to clinicians and patients on the use of TCE.

\section{METHODS}

Search strategy: The literature search will be conducted by two researchers (YQQ \& ZF) independently in the form of "back-toback", discrepancies regarding included studies will be discussed and settled by a third reviewer. We will use the combination of medical subject headings(Mesh) and text words to complete the literature search strategies, mainly include: 1) "Qigong", "Qigong", "Chi gong", "Ch'i Kung", "Chi gung", 2) " Tai Ji Quan", "Tai chi", "Tai ji", "Taijiquan", 3) "Ba duanjin", "Ba duanjin qigong", "Eight Fragments", 4) "osteoarthritis, knee", "knee osteoarthritis", "Knee Osteoarthritides", " Osteoarthritis of Knee", "Osteoarthritis of the Knee",5) "clinical trial ", "randomized controlled trial". Besides, we will scan the reference lists of included original or relevant reviews to identify additional relevant articles.

Participant or population: Studies with adults (18 years of age and older) of mild to moderate knee osteoarthritis (KOA) will be enrolled. Subjects with knee trauma or surgery, and/or history of rheumatoid arthritis will be excluded. Studies of patients with cognitive impairment will be also excluded. The clinical diagnosis of KOA should fulfill the internationally recognized criteria, diagnosed through the use of knee medical records or physical examination or laboratory/ radiographs evidence, such as the Classification Criteria of the American College of Rheumatology.

Intervention: A comparison of monotherapy of TCE with other treatments will be included. TCE plus another intervention versus the same intervention alone (e. g, TCE and NSAIDs versus only NSAIDs) will be also enrolled. No restrictions were made regarding details of this protocol, such as exercise version, training periods, and duration.

Comparator: The control group should adopt one of the following treatment methods: a placebo, no treatment, usual care, or guideline-recommended conventional treatment. If the control group contains other non-conventional therapies, such as herbal medicine, acupuncture, moxibustion, massage, yoga, will be excluded.

Study designs to be included: Only randomized controlled trials (RCTs) including combination therapy and monotherapy of TCE (Qigong, Tai Chi, Ba duanjin) will be included.

Eligibility criteria: Only RCTs will be included, in which the intervention group must have a form of TCE, while the control group can involve a placebo or no treatment. The potential outcome measures will include pain scores(e. $\mathrm{g}$,WOMAC,VAS), physical function of joint, quality of life and adverse events.

Information sources: MEDLINE, EMBASE, Web of Science, the Cochrane Central Register of Controlled Trials, the Chinese Biomedical Literature Database, ClinicalTrials.gov and the Chinese Clinical Trial Registry Platform, will be searched 
from database inception to August 20th 2020. We will contact the original investigators for more complete details of the study to solve questions about eligibility if necessary.Besides, we will scan the reference lists of included studies or relevant reviews to identify additional eligible studies.

Main outcome(s): The total score of the Western Ontario and McMaster Universities Osteoarthritis Index (WOMAC), separately addressing the severity of pain (5 questions) and any limitation in physical function(17 questions) for the activities of daily living during the past 48 hours; Pain intensity was measured on the Visual Analogue Score (VAS). For graphical information, numerical data were extracted using a standard procedure.

Additional outcome(s): Physical function was measured on the Knee injury and Osteoarthritis Outcome Score-Physical Function Short Form(KOOS-PS) or the WOMAC physical function scale; Quality of life was furthermore assessed by using the Short Form-36 Health Survey (SF-36), which is a validated tool for measuring general health. Safety was evaluated by monitoring adverse events during the study or the number of drop-outs due to health-related problems.

Data management: Two researchers (Qingqing Ye \& Lin Liu) screen titles and abstracts of retrieved studies independently to exclude obviously irrelevant studies. Full texts of the potential studies will be then reviewed to determine eligible trials. Data will be extracted on study characteristics such as basic information (date of publication, country), participants (sample size, mean age, gender, duration of symptom), methodological description, intervention characteristics, duration of treatment, frequency, outcome data, conclusion, adverse events and follow-up assessment. If the data is incomplete, the original author will be contacted. During this process, the third reviewer (Feng Zhang) will be responsible for verifying the data extracted by the two reviewers. Inconsistencies will be resolved by discussion, and consulting the superior expert (Bing Liu) to facilitate the decision when a disagreement persisting. The Preferred Reporting Items for Systematic Review and Meta-analysis (PRISMA) flow chart will be provided to explain the basic framework of process of extraction for directing the reviewers.

Quality assessment / Risk of bias analysis: Two researchers (Qing-qing $Y e$ and Fei Yang), trained before this review to ensure consistency between them, will assess the methodological quality and the risk of bias of all related studies in a blinded fashion according to a collaboration tool based on the Cochrane Handbook (version 5.3.0). The risks of bias, including 7 components as random sequence generation, allocation concealment, blinding of outcome assessment, blinding of participants and personnel, incomplete outcome data, selective outcome reporting, and other bias, will be rated as low, unclear, or high. Disagreements will be discussed and resolved for consensus.

Strategy of data synthesis: Review Manager 5.3 software, provided by the Cochrane Collaboration (www.cochrane.org), will be used for data analysis. We'll chose the random-effects model to combine all summary outcome measures. Continuous outcomes will be presented as standardized mean difference (SMD)with 95\% confidence interval (CI) between groups. An SMD of 0.20 is considered a small difference between the experimental and the control group; 0.50, a moderate difference; and 0.80 , a large difference. Dichotomous data will be analyzed using relative risk (RR) with $95 \%$ $\mathrm{Cl}$. If possible, the interpolation method will be used for the missing data after estimating the important data carefully.

Subgroup analysis: Subgroup analysis will be further stratified by type of treatment (Qi gong, Tai chi, Ba duanjin), type of control treatment(no treatment, usual care, placebo or any guideline-recommended conventional treatment), intervention duration or co-interventions. 
Sensibility analysis: Sensitivity analysis will be performed by removing the inclusion of studies one by one, for evaluating whether the results of meta-analysis is reliable and finding the source of heterogeneity.

Language: Only studies reported in English or Chinese language will be included.

Country(ies) involved: China.

Keywords: Knee osteoarthritis, traditional Chinese exercise, protocol, systematic review.

Dissemination plans: the results will be published in a scientific journal after peerreview and disseminated electronically or in print.

Contributions of each author:

Author 1 - Qing-qing Ye designed the study protocol and drafted the manuscript.

Author 2 - Lin Liu - wrote together and refined the manuscript.

Author 3 - Feng Zhang is resposible for data extraction.

Author 4 - Fei Yang is resposible for data analysis and review.

Author 5 - Xiao-yan Shi is responsible for related software.

Author 6 - Bing Liu revised and finalized the study protocol. 